\title{
The Prognostic Role of Aortic Stiffness in Patients Hospitalized for an Acute Heart Failure Syndrome
}

\author{
Sophia Giannitsi ${ }^{1},\left(\mathbb{D}\right.$, Mara Bougiakli $^{1}$, Aris Bechlioulis ${ }^{1},(\mathbb{D})$, Anna Kotsia ${ }^{1}$, Lampros Lakkas $^{1}$, Ioannis Girdis ${ }^{1,(\mathbb{D}}$, \\ Konstantinos Pappas ${ }^{1}$, Georgios Chasiotis ${ }^{2, \mathbb{D}}$, Eleni Bairaktari', (D), Andreas Kalogeropoulos ${ }^{3, \mathbb{D}}$, \\ Lampros K. Michalis ${ }^{1,(D)}$, Katerina K. Naka ${ }^{1, *}$, (i)
}

${ }^{1}$ 2nd Department of Cardiology and Michaelidion Cardiac Center, University of Ioannina, Ioannina, Greece

${ }^{2}$ Laboratory of Clinical Chemistry, University Hospital of Ioannina, Ioannina, Greece

${ }^{3}$ Department of Cardiology, University of Patras, Patras, Greece

\section{ARTICLE INFO}

\section{Article History}

Received 14 June 2020

Accepted 23 September 2020

Keywords

Acute heart failure

prognosis

aortic stiffness

pulse wave velocity

pulsatile hemodynamics

\begin{abstract}
Background: Although impaired arterial function has been associated with adverse prognosis in chronic Heart Failure (HF), its role in Acute HF Syndromes (AHFS) has been little studied. We prospectively investigated the prognostic role of arterial function on mortality and HF Hospitalizations (HHF) in patients with AHFS.

Design and Methods: A thorough assessment of arterial function was performed in patients hospitalized for AHFS $24-48 \mathrm{~h}$ before discharge and followed-up for 6 months for all-cause death and HHF. MAGGIC risk score was used to evaluate the additive predictive value of vascular biomarkers for clinical events.

Results: One-hundred patients were studied; aged $70 \pm 11$ years, $78 \%$ males, $61 \%$ had left ventricular ejection fraction $\leq 40 \%$ and $24 \% \geq 50 \%$. Mean aortic Pulse Wave Velocity (PWV) was $11.2 \mathrm{~m} / \mathrm{s}$, mean augmentation index $21 \%$ and median brachial flowmediated dilation 3.14\%. Higher PWV was associated with all-cause mortality (Hazard Ratio [HR] 1.32 per $1 \mathrm{~m} / \mathrm{s}, p<0.001$ ) and the combined clinical event of mortality and HHF (HR 1.12 per $1 \mathrm{~m} / \mathrm{s}, p=0.012$ ) even after adjustment for MAGGIC score. MAGGIC score predicted mortality (HR 3.40 per group increase, Area under Curve $[\mathrm{AUC}]=0.741, p=0.017$ ) in our population; addition of PWV to MAGGIC score increased the predictive accuracy ( $\mathrm{AUC}=0.911, \mathrm{C}$-statistic $p<0.01$ vs. MAGGIC score alone) for mortality.

Conclusion: In these AHFS patients, increased aortic stiffness was independently associated with mortality and further improved the predictive accuracy of an established risk model. Further research is needed to show whether a comprehensive assessment of AHFS patients focusing both on cardiac and vascular function, may improve management and ameliorate prognosis following an AHF hospitalization.
\end{abstract}

\section{HIGHLIGHTS}

- The interaction between the heart and the arteries is a determinant of cardiovascular function.

- Increased aortic Pulse Wave Velocity (PWV) predicts mortality in acute heart failure.

- Aortic PWV increases predictive accuracy of MAGGIC score.

(C) 2020 Association for Research into Arterial Structure and Physiology. Publishing services by Atlantis Press International B.V. This is an open access article distributed under the CC BY-NC 4.0 license (http://creativecommons.org/licenses/by-nc/4.0/).

\section{INTRODUCTION}

Heart Failure (HF) is a modern epidemic leading to increased mortality and impaired quality of life [1]. Acute Heart Failure Syndromes (AHFS), either in newly diagnosed HF or acutely decompensated chronic HF patients, are a common cause of hospitalization. Frequent Hospitalizations for $\mathrm{HF}(\mathrm{HHF})$ increase cost and portend a worse prognosis for patients as they indicate a deterioration of their health status [2]. There have been several efforts to identify contributors to

“Corresponding author. Email: drkknaka@gmail.com; anaka@uoi.gr

Peer review under responsibility of the Association for Research into Arterial Structure and Physiology

Data availability statement: The data that support the findings of this study are available from the corresponding author, Dr Katerina K. Naka, upon reasonable request. rehospitalization after HHF in order to identify patients at increased risk and implement prevention strategies [3-5].

Arterial function, as assessed by measurement of endotheliumdependent vasodilation, aortic Pulse Wave Velocity (PWV), central aortic pressures and arterial elasticity indices, has been associated with atherosclerotic disease progression and cardiovascular events, including HHF, in various populations [6-9]. In patients with chronic HF, arterial function indices have been found to be impaired either as a result of advanced atherosclerosis or HF per se $[10,11]$, leading to impaired ventricular-arterial coupling [12] that may be related to adverse prognosis [13-16].

Acute heart failure syndromes are associated with acute hemodynamic changes that may affect vascular function, while various 
vasoactive treatments administered during the acute phase could also affect arterial function. Few studies have addressed the role of on-admission [17,18] and pre-discharge [19] arterial function on risk stratification after an AHFS. Furthermore, the relative role of each marker (i.e. aortic stiffness, peripheral arterial elasticity, endothelial function etc.) in AHFS prognosis is not known.

The aim of the current study was to prospectively investigate the prognostic role of pre-discharge arterial function indices assessment for 6-month all-cause mortality and HHF in patients hospitalized for an AHFS.

\section{MATERIALS AND METHODS}

\subsection{Study Population and Design}

We prospectively studied consecutive patients who were hospitalized due to AHFS diagnosed based on the criteria established in current guidelines of the European society of cardiology [20]. All patients aged $\geq 18$ years old were eligible for enrollment. Patients with an acute myocardial infarction that led to HF presentation or during the last 3 months, severe valvular disease, chronic atrial fibrillation, congenital heart disease or other severe chronic disease (e.g. cancer, chronic renal failure on hemodialysis etc.) were excluded. The study took place in the University Hospital of Ioannina between January 2012 and December 2013. During this period, we enrolled 100 patients that were eligible based on the above-mentioned inclusion and exclusion criteria. The study complied with the Declaration of Helsinki and all participants provided written informed consent. The study protocol was approved by the local Ethics Committee.

All patients were examined $24-48 \mathrm{~h}$ prior to discharge after having been stabilized and no longer requiring the administration of intravenous diuretics or vasoactive medications. A comprehensive medical record was recorded, and a detailed physical examination was performed. Blood samples were taken early in the morning after an overnight fasting for the determination of various metabolic parameters while a serum sample was kept frozen at $-80^{\circ} \mathrm{C}$ for the determination of Brain Natriuretic Peptide (BNP). A detailed echocardiographic study was performed along with a thorough investigation of vascular function on the same day.

\subsection{Definitions of Clinical Characteristics}

The New York Heart Association (NYHA) scale I-IV was used to assess the functional status of all patients prior to discharge. A history of ischemic cardiomyopathy was defined as the documented presence of coronary artery disease that could explain the clinical presentation of HF in the patient. The presence of hypertension, hypercholesterolaemia, diabetes mellitus and smoking was recorded in all patients. Weight and height were measured, and body mass index was calculated in all study participants. Metabolic parameters in the blood were measured by standard methodology. Serum BNP levels were assessed using chemiluminescent microparticle immunoassay (ARCHITECT BNP iSystem, Abbott Laboratories, Diagnostics Division, Abbott Park, IL, USA) (assay sensitivity $10 \mathrm{pg} / \mathrm{ml})$.

\subsection{MAGGIC Score}

The MAGGIC risk score for prediction of mortality in patients with HF [21] was constructed based on the Meta-Analysis Global Group in Chronic Heart Failure and has been shown to perform well in patients after discharge for an AHFS [22]. An integer risk score is calculated based on the assessment of 13 variables: age, Left Ventricular Ejection Fraction (LVEF), NYHA functional class, serum creatinine, diabetes, beta-blocker use, systolic blood pressure, body mass index, time since diagnosis of HF, current smoker, chronic obstructive pulmonary disease, gender, and angiotensinconverting enzyme inhibitor or angiotensin receptor blockers use, and the mortality risk was classified in six groups in the original publication [21]. Currently we use a 3-scale classification of the MAGGIC score i.e. A (groups 1 and 2, score $\leq 20$ ), B (groups 3 and 4 , score 21-28) and C (groups 5 and 6, score $\geq 29$ ).

\subsection{Vascular Measurements}

Vascular studies were performed as previously described $[23,24]$ in all participants and were performed by two skilled and experienced operators $(\mathrm{SG}, \mathrm{MB})$. All exams took place in the morning between 08.00 and $10.00 \mathrm{am}$.

\subsubsection{Endothelial function}

Endothelial function was assessed by measurement of endothelium dependent Flow-mediated Dilation (FMD) of the brachial artery, according to the published guidelines [25]. Optimal images of the brachial artery and Doppler indices were obtained using a Vivid I GE Healthcare (IL, US) ultrasound machine. All images were recorded at end-diastole coincident with the $R$-wave on the electrocardiogram and stored for offline analysis. The brachial artery was occluded for $5 \mathrm{~min}$ using a wrist cuff inflated to $300 \mathrm{mmHg}$ causing an ischemic stimulus that resulted in brachial artery hyperemia. FMD was calculated as a percent increase in diameter during hyperemia compared with the brachial diameter at rest. The operators recorded the artery in a perpendicular plane so that the intima-media margins were visible both in the proximal and distal vessel wall if possible. Furthermore, the flow in the brachial artery immediately after the release of the cuff was recorded in order to make sure that an adequate increase in blood flow was achieved.

\subsubsection{Arterial stiffness}

Aortic stiffness was assessed non-invasively by measurement of aortic PWV using applanation tonometry.

Carotid-femoral PWV was estimated using the Sphygmocor system (Version 7.01, AtCor Medical, Sydney, Australia). Pressure waveforms were recorded from the carotid and femoral arteries, mostly the right ones. The distance traveled by the pulse wave was measured over the body surface as the distance between the suprasternal notch and femoral artery subtracting the distance from suprasternal notch to the carotid artery. The wave transit time between the two sites was calculated using as reference frame the $R$-wave on the simultaneously recorded electrocardiogram. 
PWV was calculated as the ratio distance/transit time in $\mathrm{m} / \mathrm{s}$. $\mathrm{PWV}>10 \mathrm{~m} / \mathrm{s}$ is considered abnormal [26].

\subsubsection{Pulsatile hemodynamics}

Augmentation Index (Alx), Central Pressures and peripheral arterial compliance were assessed non-invasively using applanation tonometry.

Augmentation index was extracted by the pressure waveform acquired from the right radial artery using the Sphygmocor system (Version 7.01, AtCor Medical). After 15-20 waveforms were acquired, the software generated the corresponding central aortic pressure waveform using a generalized transfer function. Alx was calculated as the difference between the second and first systolic peaks of the central pulse waveform. Alx was expressed as a percentage of the central pulse pressure and was finally corrected for heart rate (Alx@75). Pulse pressure was defined as systolic minus diastolic pressure. All measurement that had a quality index provided by Sphygmocor $>80$, were stored for further analysis while other measurements were rejected.

The Large Artery Elasticity Index (LAEI) and Small Artery Elasticity Index (SAEI) artery elasticity index were calculated using the HDI/Pulsewave CR-2000 Cardiovascular Profiling System (Hypertension Diagnostic, Eagan, MN, USA) as previously described [27]. A suitably sized blood pressure cuff was placed around the patient's left arm for simultaneous blood pressure recordings. A rigid, plastic wrist stabilizer was placed on the right wrist in order to achieve stability during the recording of pulse waveform. This methodology uses a modified Windkessel model to derive arterial compliance of proximal (LAEI) and distal (SAEI) arteries, by analyzing the diastolic portion of the pulse pressure contour. Abnormal values of both indices according to age and gender have been previously published [27]. Measurements with a Relative Strength Index $>15 \%$ (provided by the HDI/Pulsewave CR-2000 Cardiovascular Profiling System) were stored for further analysis while other measurements were rejected.

Reproducibility analysis of vascular studies has been previously published [23]. In studies performed on two separate days (7-10 days apart) in 10 subjects by a single operator, the within-subject coefficient of variation of FMD, PWV, LAEI and SAEI were 6.9\%, $5.6 \%, 6.8 \%$ and $9.1 \%$ respectively.

\subsection{Echocardiography Measurements}

A detailed two-dimensional and Doppler transthoracic echocardiogram was performed by an experienced operator, using a Vivid I, GE Healthcare (IL, US) machine. Classical echocardiographic indices of Left Ventricular (LV) systolic and diastolic function were recorded. LVEF was measured using the biplane Simpson's method. Based on this, patients were classified as Heart Failure with Reduced Ejection Fraction (HFrEF, i.e. LVEF $\leq 40 \%$ ), Heart Failure with mid-range Ejection Fraction (HFmrEF, LVEF $>40 \%$ and $<50 \%$ ) or Heart Failure with preserved Ejection Fraction (HFpEF, i.e. LVEF $\geq 50 \%$ ). Left atrial volume index was used as a measure of left atrial size. Tissue Doppler imaging was used to assess the early diastolic velocity of the septal and lateral mitral annulus ( $E^{\prime}$ average) in order to further calculate the $E / E^{\prime}$ ratio as a measure of LV filling pressures and diastolic function.

\subsection{Follow-up and Outcomes}

All participants were followed-up at 6 months to record any clinical events; a telephone interview of the patients who could not attend the 6-month follow-up (or their relatives) was performed. Pre-specified clinical events (death and new HF hospitalizations) were confirmed through medical records or interviews with physicians.

\subsection{Statistical Analysis}

Continuous data are presented as median (interquartile range) for not normally distributed variables and mean \pm SD for normally distributed variables. Cox regression analysis was used to identify associations between clinical outcomes at 6-month follow-up and vascular parameters, BNP and MAGGIC score. Multivariate Cox regression analysis was used to adjust the association of PWV with clinical events for MAGGIC score. The area under the curve of regression models for the prediction of mortality including MAGGIC score alone and MAGGIC score and PWV were calculated, and their predictive accuracy was compared using the methodology described by Hanley and McNeil (C-statistic) [28] $p$-Values were always two-sided and a value of $p<0.05$ was considered significant. The SPSS statistical software package (IBM SPSS Statistics, Version 23, NY, US) was used.

\section{RESULTS}

The baseline characteristics of the study population $(n=100)$ are presented in Table 1 . The mean age was 70 years, $78 \%$ were male, $37 \%$ had diabetes, $54 \%$ had impaired renal function [Estimated Glomerular Filtration Rate (eGFR) $<60 \mathrm{ml} / \mathrm{min} / 1.73 \mathrm{~m}^{2}$ ), and $53 \%$ had ischemic cardiomyopathy. Most patients (61\%) had LVEF $\leq 40 \%$, while $24 \%$ had LVEF $\geq 50 \%$. Median length of stay was 8 days. Pre-discharge metabolic and other biochemical parameters of the population are shown in Table 1. Prior to discharge, most patients (78\%) were on NYHA class I and II. Median BNP value was $436 \mathrm{ng} / \mathrm{ml}$. Standard pre-discharge echocardiographic parameters are shown in Table 2. Analysis of vascular biomarkers prior to discharge showed a PWV $>10 \mathrm{~m} / \mathrm{s}$, an abnormal LAEI and an abnormal SAEI in $8 \%$ and $68 \%$ of the patients respectively. Mean AIx was 21\% and median FMD was 3.14\% (Table 2).

All patients were followed-up according to study protocol. At 6-month follow-up, $32 \%$ of the study population presented a pre-specified clinical event; $9 \%$ of the patients died, while $23 \%$ had a HHF. MAGGIC risk score (as a 3-scale variable) was assessed in all patients and deaths were recorded in $0 \%, 11 \%$ and $20 \%$ in MAGGIC groups A-C respectively. MAGGIC score was associated with mortality at 6 months [HR 3.40 per group increase (95\% CI 1.25, 9.26), $p=0.017$ ], but not with HHF [HR 1.30 per group increase $(95 \% \mathrm{CI}$ $0.82,2.07), p=0.266]$ or the combined event of mortality and HHF [HR 0.91 per group increase (95\% CI 0.52, 1.60), $p=0.749$ ]. BNP levels were not related to any of the studied clinical events. The associations of vascular parameters with clinical events at follow-up in 
Table 1 Descriptive characteristics, metabolic and biochemical parameters of the study population $(n=100)$

\begin{tabular}{|c|c|}
\hline Age, years & $70 \pm 11$ \\
\hline Male gender, $n$ & 78 \\
\hline History of dyslipidemia, $n$ & 61 \\
\hline History of hypertension, $n$ & 85 \\
\hline History of diabetes, $n$ & 37 \\
\hline Current smoking, $n$ & 28 \\
\hline Chronic kidney disease, $n^{*}$ & 54 \\
\hline Anemia, $n^{* *}$ & 37 \\
\hline Chronic pulmonary disease, $n$ & 16 \\
\hline Ischemic cardiomyopathy, $n$ & 53 \\
\hline Paroxysmal atrial fibrillation, $n$ & 23 \\
\hline HFpEF (LVEF $\geq 50 \%), n$ & 24 \\
\hline HFrEF (LVEF $\leq 40 \%), n$ & 61 \\
\hline Previous decompensations within 12 months, $n$ & 21 \\
\hline \multicolumn{2}{|l|}{ Medications on admission } \\
\hline Beta blockers & 60 \\
\hline ACE-I/ARB & 57 \\
\hline Aldosterone antagonists & 34 \\
\hline Furosemide & 53 \\
\hline Digoxin & 4 \\
\hline Amiodarone & 11 \\
\hline Statin & 46 \\
\hline Body mass index, $\mathrm{kg} / \mathrm{m}^{2}$ & $27.5 \pm 4.4$ \\
\hline Estimated glomerular filtration rate, $\mathrm{ml} / \mathrm{min} / 1.73 \mathrm{~m}^{2}$ & $58.7 \pm 17.9$ \\
\hline Hemoglobin, g/dl & $13.3 \pm 1.9$ \\
\hline Fasting glucose, $\mathrm{mg} / \mathrm{dl}$ & $137(97,191)$ \\
\hline Urea, $\mathrm{mg} / \mathrm{dl}$ & $57(42,78)$ \\
\hline Sodium, mmol/1 & $138 \pm 3$ \\
\hline Potassium, mmol/l & $4.3 \pm 0.6$ \\
\hline Uric acid, mg/dl & $7.5 \pm 2.3$ \\
\hline Total cholesterol, mg/dl & $166 \pm 38$ \\
\hline High density lipoprotein cholesterol, mg/dl & $40 \pm 10$ \\
\hline Low density lipoprotein cholesterol, mg/dl & $99 \pm 31$ \\
\hline Triglycerides, mg/dl & $120(90,140)$ \\
\hline Albumin, $\mathrm{mg} / \mathrm{dl}$ & $3.9(3.6,4.2)$ \\
\hline Brain natriuretic peptide, $\mathrm{ng} / \mathrm{ml}$ & $436(110,832)$ \\
\hline
\end{tabular}

univariate analysis are shown in Table 3. PWV was associated with mortality (HR 1.32 per $1 \mathrm{~m} / \mathrm{s}$ increase, $p<0.001$ ) and the combined event of mortality and HF re-hospitalizations (HR 1.12 per $1 \mathrm{~m} / \mathrm{s}$ increase, $p=0.014$ ) at 6 months. After adjustment for MAGGIC score, PWV remained an independent predictor of mortality [HR 1.38 per $1 \mathrm{~m} / \mathrm{s}$ increase $(95 \%$ CI $1.19,1.63), p<0.001$ ] and the combined event of mortality and HHF [HR 1.12 per $1 \mathrm{~m} / \mathrm{s}$ increase $(95 \%$ CI $1.03,1.22), p=0.012]$. None of the other studied vascular markers was related to any of the clinical events at follow-up (Table 3). In Receiver Operating Characteristic (ROC) curve analysis, MAGGIC risk score predicted mortality with an $\mathrm{AUC}=0.741, p=0.017)$. The addition of PWV to MAGGIC score resulted in AUC $=0.911, p<$ 0.001 ( $p<0.01$ vs. MAGGIC score alone) (Figure 1 ).

\section{DISCUSSION}

In the present study, arterial function indices were assessed in patients hospitalized with AHFS prior to discharge. Aortic PWV,
Table 2 Vascular and echocardiographic parameters of the study population prior to discharge $(n=100)$

\begin{tabular}{lc}
\hline Systolic blood pressure, $\mathrm{mmHg}$ & $123 \pm 20$ \\
Diastolic blood pressure, mmHg & $72 \pm 10$ \\
Heart rate, beats per minute & $69(60,79)$ \\
Pulse wave velocity, $\mathrm{m} / \mathrm{s}$ & $11.2 \pm 3.4$ \\
Pulse wave velocity $>10 \mathrm{~m} / \mathrm{s}, n$ & 57 \\
Augmentation index, \% & $21(17,29)$ \\
Aortic pulse pressure, $\mathrm{mmHg}$ & $37(29,51)$ \\
Flow-mediated dilation, $\%$ & $3.14(2.37,3.70)$ \\
Large artery elasticity index, $\mathrm{ml} / \mathrm{mmHg}^{*} 10$ & $11.2(8.0,16.2)$ \\
Abnormal large artery elasticity index, $n$ & 8 \\
Small artery elasticity index, ml/mmHg ${ }^{*} 100$ & $3.4(2.5,4.8)$ \\
Abnormal small artery elasticity index, $n$ & 68 \\
Left ventricular end-diastolic diameter, mm & $57 \pm 10$ \\
Left ventricular mass index, gr/m ${ }^{2}$ & $137 \pm 40$ \\
Increased left ventricular mass, $n^{*}$ & 71 \\
Left atrial volume index, ml/m ${ }^{2}$ & $44.8(34.0,54.0)$ \\
E/ $E^{\prime}$ & $12.6(9.0,18.6)$ \\
E/E' $>14$ & 40 \\
Left ventricle ejection fraction, $\%$ & $35(27,45)$ \\
Pulmonary artery systolic pressure, $\mathrm{mmHg}$ & $35(20,42)$ \\
Pulmonary artery systolic pressure $>35 \mathrm{mmHg}$ & 43 \\
Inferior vena cava diameter, mm & $15(11,20)$ \\
\hline
\end{tabular}

"Increased left ventricular mass defined as left ventricular mass index $>95$ and $115 \mathrm{~g} / \mathrm{m}^{2}$ for women and men respectively. Continuous variables are presented as mean \pm SD or median (interquartile range). $E / E^{\prime}$, early diastolic mitral inflow velocity to mitral annular early diastolic velocity ratio.

an index of aortic stiffness, was independently associated with the occurrence of death and HHF at 6-months.

Our population consisted of HF patients with both reduced and preserved LVEF in a relatively stable condition pre-discharge, mostly in functional class NYHA I and II with residual congestion (as indicated by increased BNP and echocardiographic findings of increased $E / E^{\prime}$ ratio $>14$ and systolic pulmonary artery pressure $>35 \mathrm{mmHg}$ in $>40 \%$ of the patients). Patients presented increased aortic stiffness (i.e. PWV $>10 \mathrm{~m} / \mathrm{s}$ ), abnormal small artery distensibility and severely impaired endothelial function (i.e. median brachial FMD ca. 3.0\%) in the majority of our population. Impairment of arterial function indices in HF patients has been attributed to increased age, high prevalence of cardiovascular risk factors (i.e. hypertension, diabetes) and coronary artery disease [11,29], characteristics also found in our population. Aortic stiffness may accelerate $\mathrm{HF}$ decompensation in vulnerable patients by increasing systolic load and worsening ventricular-vascular coupling. Excessive neurohormonal activation and inflammation as part of the HF syndrome itself, as occurring in AHFS, may further aggravate arterial function [30-32], while following the acute phase and transitioning to chronic HF, arterial stiffness may decrease [31]. Volume retention causes perturbations of pulsatile haemodynamics, manifested by an abrupt increase in arterial stiffness and blood pressure that acutely impair LV diastolic dysfunction leading to an AHFS [33,34]. This may be the rationale for vasodilator therapy in AHFS [35].

Currently, we demonstrated that in AHFS patients, increased aortic PWV pre-discharge was independently associated with increased risk for mortality or the combined clinical endpoint of mortality and HHF. Furthermore, adding PWV to MAGGIC score, a risk score for mortality in chronic HF patients [21] which has been also validated in patients after AHFS [22], increases considerably 
Table 3 Univariate associations of vascular parameters with clinical events at follow-up

\begin{tabular}{|c|c|}
\hline \multicolumn{2}{|c|}{ Mortality and hospitalization for heart failure at 6 months } \\
\hline Systolic BP per 10 mmHg increase & HR $1.15(95 \%$ CI 0.97, 1.37), $p=0.097$ \\
\hline Diastolic BP per $10 \mathrm{mmHg}$ increase & HR $1.38(95 \%$ CI $0.98,1.96), p=0.066$ \\
\hline PWV per $1 \mathrm{~m} / \mathrm{s}$ & HR $1.12(95 \%$ CI 1.02, 1.22$), p=0.014$ \\
\hline AIx per $5 \%$ increase & HR $1.04(95 \%$ CI $0.87,1.25), p=0.653$ \\
\hline cPP per $5 \mathrm{mmHg}$ & HR $1.10(95 \%$ CI $0.99,1.22), p=0.072$ \\
\hline FMD per $1 \%$ increase & HR $1.03(95 \%$ CI $0.77,1.38), p=0.849$ \\
\hline LAEI per $5 \mathrm{ml} / \mathrm{mmHg}{ }^{*} 10$ increase & HR 0.97 (95\% CI $0.76,1.24), p=0.818$ \\
\hline SAEI per $2 \mathrm{ml} / \mathrm{mmHg}^{\star} 100$ increase & HR $0.92(95 \%$ CI $0.67,1.25), p=0.575$ \\
\hline \multicolumn{2}{|l|}{ Mortality at 6 months } \\
\hline Systolic BP per $10 \mathrm{mmHg}$ increase & HR 1.07 (95\% CI 0.78, 1.46), $p=0.683$ \\
\hline Diastolic BP per $10 \mathrm{mmHg}$ increase & HR 1.10 (95\% CI 0.58, 2.08), $p=0.781$ \\
\hline PWV per $1 \mathrm{~m} / \mathrm{s}$ & HR $1.32(95 \%$ CI 1.15, 1.53$), p<0.001$ \\
\hline AIx per $5 \%$ increase & HR 0.85 (95\% CI $0.58,1.23), p=0.384$ \\
\hline cPP per $5 \mathrm{mmHg}$ & HR 1.01 (95\% CI 0.83, 1.23), $p=0.944$ \\
\hline FMD per $1 \%$ increase & HR $0.56(95 \%$ CI $0.27,1.18), p=0.127$ \\
\hline LAEI per $5 \mathrm{ml} / \mathrm{mmHg}{ }^{*} 10$ increase & HR $0.81(95 \%$ CI $0.47,1.41), p=0.461$ \\
\hline SAEI per $2 \mathrm{ml} / \mathrm{mmHg}^{*} 100$ increase & HR $0.79(95 \%$ CI $0.39,1.62), p=0.522$ \\
\hline \multicolumn{2}{|l|}{ Hospitalization for heart failure at 6 months } \\
\hline Systolic BP per 10 mmHg increase & HR 1.17 (95\% CI 0.96, 1.43), $p=0.115$ \\
\hline Diastolic BP per $10 \mathrm{mmHg}$ increase & HR $1.44(95 \%$ CI $0.95,2.16), p=0.083$ \\
\hline PWV per $1 \mathrm{~m} / \mathrm{s}$ & HR $1.05(95 \%$ CI $0.94,1.18), p=0.483$ \\
\hline AIx per $5 \%$ increase & HR $1.04(95 \%$ CI $0.84,1.29), p=0.704$ \\
\hline cPP per $5 \mathrm{mmHg}$ & HR $1.11(95 \%$ CI $0.98,1.25), p=0.095$ \\
\hline FMD per $1 \%$ increase & HR $1.09(95 \%$ CI $0.79,1.52), p=0.599$ \\
\hline LAEI per $5 \mathrm{ml} / \mathrm{mmHg}$ * 10 increase & HR $1.02(95 \%$ CI $0.77,1.35), p=0.892$ \\
\hline SAEI per $2 \mathrm{ml} / \mathrm{mmHg}{ }^{\star} 100$ increase & HR 0.87 (95\% CI $0.59,1.28), p=0.467$ \\
\hline
\end{tabular}

BP, blood pressure; CI, confidence interval; cPP, central aortic pulse pressure; HR, hazard ratio; Ln, natural logarithm.

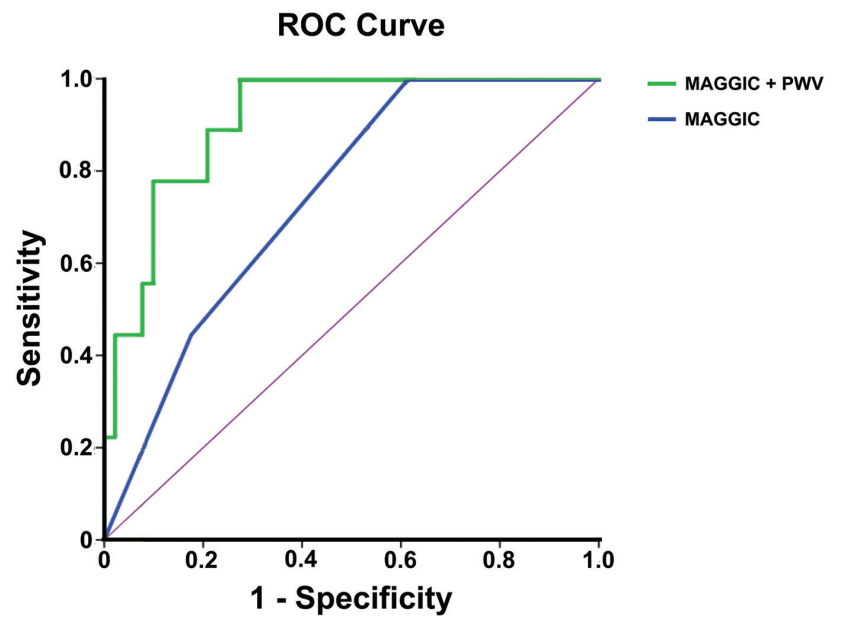

Figure 1 Receiver operating curves for the two models, i.e. MAGGIC score only and MAGGIC score plus pulse wave velocity, for the prediction of mortality at 6 months.

the prognostic accuracy of the combined model for mortality at 6 months. However, our results must be interpreted with caution because the absolute number of events was small. In addition, this is the first study to show a prognostic role of MAGGIC risk score for mortality in short-term (i.e. 6-months follow-up); previous studies have validated the prognostic role of the risk score for 1-3 years follow-up $[21,22]$. Pre-discharge aortic PWV has been previously shown to be an independent predictor of mortality following a HHF $[19,36]$. On-admission PWV (in patients hospitalized with AHFS) has been also associated with post-discharge adverse outcomes, but this association was lost after adjustment for age, eGFR, hemoglobin, and natriuretic peptides [18].

Perturbations of the central aortic pulsatile hemodynamics have been suggested to be involved in the development of AHF [37], while post-discharge events commonly occur in patients with incomplete recovery of the pulsatile hemodynamics [19]. Increased aortic PP showed only a trend, although nonsignificant, to higher incidence of HHF at 6 months in our study. Increased aortic PP, assessed either on admission [18] or predischarge [19] has been independently related to worse clinical outcomes in mixed populations with AHFS (both HFrEF and HFpEF). Of note, in AHFS patients with HFrEF only, lower aortic PP has been independently associated with higher allcause mortality $[17,36]$. The role of aortic PP in patients with HFrEF is more complex as the lower systolic boost of the forward wave because of severe LV dysfunction and low stroke volume, rather than increased peripheral vascular resistance and stiffness, mainly determine aortic PP [38]. In the current study of a mixed AHFS population, aortic PP has not shown a clear predictive value probably because the conceptually anticipated effects of a lower PP in HFrEF is cancelled out but the effects of a higher PP in HFpEF. Nevertheless, larger future studies are needed to clarify these hypotheses.

Furthermore, endothelial function in our population was found to be severely impaired as assessed by the low brachial artery FMD (i.e. median value ca. 3.0\%); previous studies in chronic HF patients have demonstrated higher FMD values (i.e. mean values $>4.0-5.0 \%$ ) [14,39], probably suggesting that FMD may be further attenuated in the acute setting of HF decompensation. Indeed, impairment in $\mathrm{NO}$-dependent and independent 
pathways of endothelial function have been implicated in AHFS [40]. In chronic HF patients, endothelial dysfunction has been shown to be common [14,39] and has been associated with poor outcomes irrespective of HF etiology [14,41,42] providing incremental prognostic information in addition to BNP [42]. Nevertheless, FMD was not found to be associated with clinical outcomes probably because endothelial function was severely impaired invariably in our HF patients.

In contrast to central aortic stiffness, markers of peripheral arterial compliance have not shown a prognostic significance in our study. SAEI and LAEI indices have not been previously studied in the setting of HF. The greater prognostic importance of central aortic versus peripheral arterial elasticity indices in AHFS, implied in our study, needs to be further validated.

Interestingly, the natriuretic peptide BNP at discharge did not correlate with adverse clinical events at follow-up in our study. The exact time of measurement of natriuretic peptides (admission vs. discharge) and their change during hospitalization has been extensively studied with contradicting results in relation to prognosis [43].

\subsection{Limitations}

This was a single center study and the results may be affected by local clinical practice in the management of AHF patients. The sample size is relatively small and although incidence of clinical events was relatively high in our population ( $9 \%$ and $23 \%$ for mortality and HHF respectively at 6 months), the prognostic role of various vascular indices should be evaluated in larger studies, as the absolute number of events was small. The small sample size precluded the analysis of the predictive value of studied parameters based on Cox-regression models and hence ROC analysis was performed. The results cannot be generalized in HF patients with atrial fibrillation since these patients were excluded from the current study; vascular studies' protocols have not been validated in the presence of atrial fibrillation.

\section{CONCLUSION}

The interaction between the heart and the systemic arterial vasculature is a key determinant of cardiovascular performance and assessment of arterial function, especially central aortic stiffness, may be a valuable tool in the prediction of adverse events following an AHF hospitalization. Increased aortic stiffness (i.e. increased PWV) may predict mortality and HF re-hospitalizations in AHF patients. Measurement of aortic PWV may further increase the prognostic ability of well-known risk scores for mortality in AHF patients. Further research is needed to investigate whether a comprehensive evaluation of AHF patients, assessing both cardiac and vascular function, allowing individualized targeted management, may ameliorate prognosis following an AHF hospitalization.

\section{CONFLICTS OF INTEREST}

The authors declare they have no conflicts of interest.

\section{AUTHORS' CONTRIBUTION}

SG and KKN contributed in study conception, manuscript drafting, data-analysis and data collection. MB contributed in study conception, data-analysis and data collection. $\mathrm{AB}$ contributed in study conception, manuscript drafting, data-analysis. AK, LL, IG, $\mathrm{KP}, \mathrm{GC}$ and EB contributed in critical appraisal and data collection. AK contributed in critical appraisal and manuscript editing. LKM contributed in study conception, critical appraisal, manuscript editing.

\section{FUNDING}

This research did not receive any specific grant from funding agencies in the public, commercial, or not-for-profit sectors.

\section{REFERENCES}

[1] Savarese G, Lund LH. Global public health burden of heart failure. Card Fail Rev 2017;3:7-11.

[2] Cheema B, Ambrosy AP, Kaplan RM, Senni M, Fonarow GC, Chioncel $\mathrm{O}$, et al. Lessons learned in acute heart failure. Eur J Heart Fail 2018;20:630-41.

[3] Sliwa K, Davison BA, Mayosi BM, Damasceno A, Sani M, Ogah OS, et al. Readmission and death after an acute heart failure event: predictors and outcomes in sub-saharan africa: Results from the THESUS-HF registry. Eur Heart J 2013;34:3151-9.

[4] Thavendiranathan P, Yingchoncharoen T, Grant A, Seicean S, Landers SH, Gorodeski EZ, et al. Prediction of 30-day heart failure-specific readmission risk by echocardiographic parameters. Am J Cardiol 2014;113:335-41.

[5] Passantino A, Monitillo F, Iacoviello M, Scrutinio D. Predicting mortality in patients with acute heart failure: role of risk scores. World J Cardiol 2015;7:902-11.

[6] Mitchell GF, Hwang SJ, Vasan RS, Larson MG, Pencina MJ, Hamburg NM, et al. Arterial stiffness and cardiovascular events: the framingham heart study. Circulation 2010;121:505-11.

[7] Duprez DA, Jacobs Jr DR, Lutsey PL, Bluemke DA, Brumback LC, Polak JF, et al. Association of small artery elasticity with incident cardiovascular disease in older adults: the multi-ethnic study of atherosclerosis. Am J Epidemiol 2011;174:528-36.

[8] Tsao CW, Lyass A, Larson MG, Levy D, Hamburg NM, Vita JA, et al. Relation of central arterial stiffness to incident heart failure in the community. J Am Heart Assoc 2015;4:e002189.

[9] Aisu H, Saito M, Inaba S, Morofuji T, Takahashi K, Sumimoto $\mathrm{T}$, et al. Association of worsening arterial stiffness with incident heart failure in asymptomatic patients with cardiovascular risk factors. Hypertens Res 2017;40:173-80.

[10] Desai AS, Mitchell GF, Fang JC, Creager MA. Central aortic stiffness is increased in patients with heart failure and preserved ejection fraction. J Card Fail 2009;15:658-64.

[11] Marti CN, Gheorghiade M, Kalogeropoulos AP, Georgiopoulou VV, Quyyumi AA, Butler J. Endothelial dysfunction, arterial stiffness, and heart failure. J Am Coll Cardiol 2012;60:1455-69.

[12] Ikonomidis I, Aboyans V, Blacher J, Brodmann M, Brutsaert DL, Chirinos JA, et al. The role of ventricular-arterial coupling in cardiac disease and heart failure: Assessment, clinical implications and therapeutic interventions. A consensus document 
of the european society of cardiology working group on aorta \& peripheral vascular diseases, european association of cardiovascular imaging, and heart failure association. Eur J Heart Fail 2019;21:402-24.

[13] Meguro T, Nagatomo Y, Nagae A, Seki C, Kondou N, Shibata M, et al. Elevated arterial stiffness evaluated by brachial-ankle pulse wave velocity is deleterious for the prognosis of patients with heart failure. Circ J 2009;73:673-80.

[14] Shechter M, Matetzky S, Arad M, Feinberg MS, Freimark D. Vascular endothelial function predicts mortality risk in patients with advanced ischaemic chronic heart failure. Eur J Heart Fail 2009;11:588-93.

[15] Bonapace S, Rossi A, Cicoira M, Targher G, Valbusa F, Benetos A, et al. Increased aortic pulse wave velocity as measured by echocardiography is strongly associated with poor prognosis in patients with heart failure. J Am Soc Echocardiogr 2013;26:714-20.

[16] Demir Ş, Akpınar O, Akkus O, Nas K, Unal I, Molnar F, et al. The prognostic value of arterial stiffness in systolic heart failure. Cardiol J 2013;20:665-71.

[17] Aronson D, Burger AJ. Relation between pulse pressure and survival in patients with decompensated heart failure. Am J Cardiol 2004;93:785-8.

[18] Sung SH, Yu WC, Cheng HM, Lee CW, Lin MM, Chuang SY, et al. Excessive wave reflections on admission predict post-discharge events in patients hospitalized due to acute heart failure. Eur J Heart Fail 2012;14:1348-55.

[19] Sung SH, Yu WC, Cheng HM, Chuang SY, Wang KL, Huang CM, et al. Pulsatile hemodynamics and clinical outcomes in acute heart failure. Am J Hypertens 2011;24:775-82.

[20] Ponikowski P, Voors AA, Anker SD, Bueno H, Cleland JGF, Coats AJS, et al. 2016 ESC guidelines for the diagnosis and treatment of acute and chronic heart failure: The task force for the diagnosis and treatment of acute and chronic heart failure of the european society of cardiology (ESC) developed with the special contribution of the heart failure association (HFA) of the ESC. Eur Heart J 2016;37:2129-200.

[21] Pocock SJ, Ariti CA, McMurray JJ, Maggioni A, Kober L, Squire IB, et al. Predicting survival in heart failure: a risk score based on 39372 patients from 30 studies. Eur Heart J 2013;34:1404-13.

[22] Khanam SS, Choi E, Son JW, Lee JW, Youn YJ, Yoon J, et al. Validation of the MAGGIC (meta-analysis global group in chronic heart failure) heart failure risk score and the effect of adding natriuretic peptide for predicting mortality after discharge in hospitalized patients with heart failure. PLoS One 2018;13:e0206380.

[23] Vlahos AP, Theocharis P, Bechlioulis A, Naka KK, Vakalis K, Papamichael ND, et al. Changes in vascular function and structure in juvenile idiopathic arthritis. Arthritis Care Res (Hoboken) 2011;63:1736-44.

[24] Bechlioulis A, Vakalis K, Naka KK, Bourantas CV, Papamichael ND, Kotsia A, et al. Increased aortic pulse wave velocity is associated with the presence of angiographic coronary artery disease in overweight and obese patients. Am J Hypertens 2013;26:265-70.

[25] Thijssen DHJ, Bruno RM, van Mil ACCM, Holder SM, Faita F, Greyling A, et al. Expert consensus and evidence-based recommendations for the assessment of flow-mediated dilation in humans. Eur Heart J 2019;40:2534-47.

[26] Van Bortel LM, Laurent S, Boutouyrie P, Chowienczyk P, Cruickshank JK, De Backer T, et al. Expert consensus document on the measurement of aortic stiffness in daily practice using carotid-femoral pulse wave velocity. J Hypertens 2012;30:445-8.
[27] Zimlichman R, Shargorodsky M, Boaz M, Duprez D, Rahn KH, Rizzoni D, et al. Determination of arterial compliance using blood pressure waveform analysis with the CR-2000 system: reliability, repeatability, and establishment of normal values for healthy european population-the seven European sites study (SESS). Am J Hypertens 2005;18:65-71.

[28] Hanley JA, McNeil BJ. The meaning and use of the area under a receiver operating characteristic (ROC) curve. Radiology 1982;143:29-36.

[29] Chirinos JA, Segers P, Hughes T, Townsend R. Large-artery stiffness in health and disease: JACC state-of-the-art review. J Am Coll Cardiol 2019;74:1237-63.

[30] Ooi H, Chung W, Biolo A. Arterial stiffness and vascular load in heart failure. Congest Heart Fail 2008;14:31-6.

[31] Kim DB, Baek SH, Jang SW, Her SH, Shin DI, Park CS, et al. Improvement of arterial stiffness in the transition from acute decompensated heart failure to chronic compensated heart failure. Clin Cardiol 2013;36:358-62.

[32] Grosman-Rimon L, Billia F, Wright E, Carasso S, Elbaz-Greener G, Kachel E, et al. Neurohormones, inflammatory mediators, and cardiovascular injury in the setting of heart failure. Heart Fail Rev 2020;25:685-701.

[33] Gheorghiade M, Abraham WT, Albert NM, Greenberg BH, O'Connor CM, She L, et al. Systolic blood pressure at admission, clinical characteristics, and outcomes in patients hospitalized with acute heart failure. JAMA 2006;296:2217-26.

[34] Milo-Cotter O, Adams KF, O’Connor CM, Uriel N, Kaluski E, Felker GM, et al. Acute heart failure associated with high admission blood pressure-a distinct vascular disorder? Eur J Heart Fail 2007;9:178-83.

[35] Gheorghiade M, Pang PS. Acute heart failure syndromes. J Am Coll Cardiol 2009;53:557-73.

[36] Regnault V, Lagrange J, Pizard A, Safar ME, Fay R, Pitt B, et al. Opposite predictive value of pulse pressure and aortic pulse wave velocity on heart failure with reduced left ventricular ejection fraction: insights from an eplerenone post-acute myocardial infarction heart failure efficacy and survival study (EPHESUS) substudy. Hypertension 2014;63:105-11.

[37] Weber T, Chirinos JA. Pulsatile arterial haemodynamics in heart failure. Eur Heart J 2018;39:3847-54.

[38] Naka KK, Ikonomidis I. Brachial pulse pressure in heart failure: simple to measure but complex to interpret. Eur Heart J 2019;40:e8-e10.

[39] Takishima I, Nakamura T, Hirano M, Kitta Y, Kobayashi T, Fujioka $\mathrm{D}$, et al. Predictive value of serial assessment of endothelial function in chronic heart failure. Int J Cardiol 2012;158:417-22.

[40] Colombo PC, Onat D, Sabbah HN. Acute heart failure as "acute endothelitis"-interaction of fluid overload and endothelial dysfunction. Eur J Heart Fail 2008;10:170-5.

[41] Katz SD, Hryniewicz K, Hriljac I, Balidemaj K, Dimayuga C, Hudaihed A, et al. Vascular endothelial dysfunction and mortality risk in patients with chronic heart failure. Circulation 2005;111:310-14.

[42] Meyer B, Mortl D, Strecker K, Hulsmann M, Kulemann V, Neunteufl T, et al. Flow-mediated vasodilation predicts outcome in patients with chronic heart failure: comparison with B-type natriuretic peptide. J Am Coll Cardiol 2005;46:1011-18.

[43] Santaguida PL, Don-Wauchope AC, Oremus M, McKelvie R, Ali U, Hill SA, et al. BNP and NT-proBNP as prognostic markers in persons with acute decompensated heart failure: a systematic review. Heart Fail Rev 2014;19:453-70. 